\title{
Excitations of a low-dimensional dimerized spin ladder under a magnetic field.
}

\author{
Sujit Sarkar \\ PornaPrajna Institute of Scientific Research, 4 Sadashivanagar, \\ Bangalore 560080 and Department of Condensed Matter Physics, \\ Weizmann Institute of Science, Rehovot-76100, Israel
}

(Dated: August 22, 2018)

\begin{abstract}
Here we study the dimerized spin ladder with nearest-neighbor $\left(J_{1}\right)$ and next-nearest-neighbor $\left(J_{2}\right)$ anti-ferromagnetic interaction under a magnetic field. We predict the existence of different magnetization plateaus for the presence of spin-Peierls interaction on both $J_{1}$ and $J_{2}$. Magnetization plateau at $m=0$ for $J_{1}$ dimerization is spontaneous due to XY interaction, but it is absent for $J_{2}$ dimerization, only intrinsic umklapp term leads to plateau (spin gap) state for some specific values of XXZ anisotropy $(\Delta)$ and $J_{2}$. Here we predict a saturation plateau which is the classical phase of the system. There are some numerical support of our analytical approach already existing in the literature. The transition from commensurate gapped phase to incommensurate Luttinger liquid phase is the Mott- $\delta$ type of transition.

PACS numbers: 75.10.Jm-Quantized Spin Models , 75.40.Cx-Static Properties (order parameters, static susceptibilities, heat capacities, critical exponents etc) , 75.45.+j-Macroscopic Quantum Phenomena in Magnetic Systems.
\end{abstract}

\section{INTRODUCTION}

One-dimensional and quasi-one-dimensional quantum spin systems have been studied extensively for last few decades, due to many unusual and interesting findings from both experimental and theoretical sides. The four observations which make the low-dimensional spin systems particularly interesting are, (i) Haldane's conjecture for one-dimensional anti-ferromagnetic spin systems [1], (ii) the discovery of high-temperature superconductivity and its magnetic properties at low doping [2], (iii) the discovery of ladder materials [3], and (iv) response of the spin-ladder/chain systems under a magnetic field (gapped excitations, magnetization plateau, magnetization cusp, and first order phase transition) [4, 5, 6, 7, 8, 9, 10, 11, 12, 13]. It has been observed that spin-1/2 ladder systems with the railroad geometry with an even number of legs are gapped, while systems with an odd number of chains have gapless excitations [14, 15]. However, the frustrated zigzag ladder shows gapless spin liquid state or the gapped dimer state, depending on the ratio of the exchanges of the rungs to the chains [16].

However, all these above mentioned studies are in the absence of magnetic field. The situation become very interesting in presence of magnetic field. Then it is possible for an integer spin chain to be gapless with partial magnetization and a half-odd-integer spin chain to show a gap above the ground state for appropriate values of magnetic field. It has been shown by different groups $[5,6,7,[8,9]$ that the magnetization of some systems can exhibit plateaus at certain nonzero values for some finite ranges of the magnetic field. The basic criteria for the appearance of magnetization plateau can be understood from the extension of Lieb-Schultz-Mattis theorem under a magnetic field. This implies that translationally invariant spin chains in an applied field can be gapped without breaking translation symmetry under the condition $S-m=$ integer, where $S$ is the spin and $m$ is the magnetization state of the chain. In this gapped phase, magnetization plateau occurs for quantized values of $\mathrm{m}$. Fractional quantization can also occur, if accompanied by spontaneous breaking of translational symmetry. Fractional quantization can be understood from the $S-m=$ non integer condition. In this situation system is either in the gapless low lying states or the degenerate ground state with spontaneous translational symmetry breaking in the thermodynamic limit. Suppose a system is in the spontaneously translational symmetry breaking state, the ground state should be degenerate (say q fold). In this situation ground state have q different eigenvalues of translational operator, the degenerate states can be related to the spontaneous breaking of translational symmetry to period of q sites in the thermodynamic limit. These conditions for the appearance of plateau are the necessary but not the sufficient conditions. The nature and the occurrence of the plateau also depend on the nature of the interaction present in the system 10, 11]. Apart from this interesting plateau formation,the presence of magnetic field has also other interesting effects on spin systems [12, 17, 18, 19.

In our present study, we will study the effect of magnetic field on the dimerized (both rung and leg), spin ladder. Here we predict the presence of three fractionally quantized magnetization plateaus $(m=0,1 / 4,1 / 2)$ whereas the other fractionally quantized plateaus are absent. We mainly stress on the study of leg-dimerization. [6]. Fig. 1 shows the zig-zag ladder with dimerized legs (different chain exchange). Rung dimerization is the dimerization in $J_{1}$, which has not been shown in the fig- 


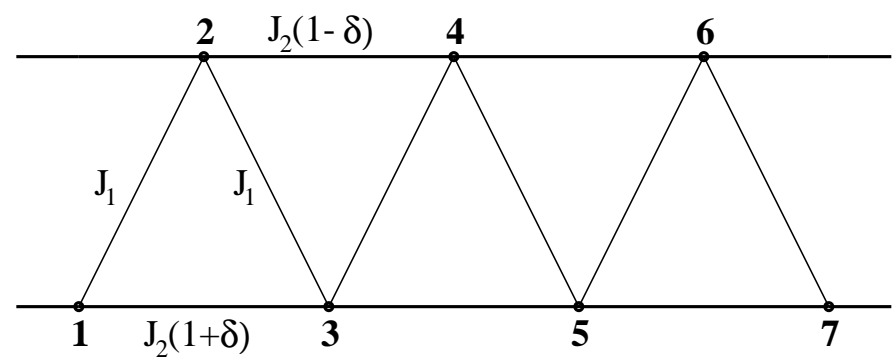

FIG. 1: Schematic diagram of a spin ladder with different chain exchanges.

ure. We compare our calculation and results at each and every steps with rung dimerization with a marginally new result in rung dimerization regarding the XXZ anisotropy of NN interaction. In this work our approach is completely analytic, we use abelian bosonization and renormalization group method to study these model Hamiltonians. We compare our results with the available numerical results. Sections of this article, are the following. Section (2). Hamiltonians and the general formulation of analytical calculations, with the analysis of different magnetization plateau. Section (3) is devoted for discussions and conclusions.

\section{MODEL HAMILTONIANS AND CONTINUUM FIELD THEORETICAL STUDY:}

In this section we will present all general derivations of this paper, different subsections for different magnetization plateaus, are the special limit of these general derivations. The model Hamiltonian for $J_{2}$ and $J_{1}$ dimerization are respectively,

$$
\begin{aligned}
H_{A}= & J_{1} \sum_{n}\left(S_{n}(x) S_{n+1}(x)+S_{n}(y) S_{n+1}(y)\right. \\
& \left.+\Delta S_{n}(z) S_{n+1}(z)\right)+J_{2} \sum_{n}\left(1-\delta(-1)^{n}\right) \vec{S}_{n} \cdot \vec{S}_{n+2} \\
& -g \mu_{B} H \sum_{n} S_{n}(z)
\end{aligned}
$$

$$
\begin{aligned}
H_{B}= & J_{1} \sum_{n}\left(1-\delta(-1)^{n}\right)\left(S_{n}(x) S_{n+1}(x)+S_{n}(y) S_{n+1}(y)\right. \\
& \left.+\Delta S_{n}(z) S_{n+1}(z)\right)+J_{2} \sum_{n} \vec{S}_{n} \cdot \vec{S}_{n+2} \\
& -g \mu_{B} H \sum_{n} S_{n}(z)
\end{aligned}
$$

Where $\mathrm{n}$ is the site index, $\mathrm{x}, \mathrm{y}$, and $\mathrm{z}$ are components of spin. One can also recast the zigzag ladder as a linear chain with NN and NNN exchange interactions [20, 21]. $J_{1}$ and $J_{2}$ are the nearest-neighbor and next-nearestneighbor exchange coupling between spins, $J_{1}, J_{2} \geq 0, \Delta$ is z component anisotropy of NN exchange interaction. $\delta$ is dimerization strength, it appear as a parameter in our Hamiltonian, the basic origin of this parameter is the spin-phonon interaction. $H$ is the externally applied magnetic field in the $\mathrm{z}$ direction. One can express spin chain systems to a spinless fermions systems through the application of Jordan-Wigner transformation. In JordanWigner transformation the relation between the spin and the electron creation and annihilation operators are

$$
\begin{aligned}
S_{n}^{z} & =\psi_{n}^{\dagger} \psi_{n}-1 / 2, \\
S_{n}^{-} & =\psi_{n} \exp \left[i \pi \sum_{j=-\infty}^{n-1} n_{j}\right], \\
S_{n}^{+} & =\psi_{n}^{\dagger} \exp \left[-i \pi \sum_{j=-\infty}^{n-1} n_{j}\right],
\end{aligned}
$$

where $n_{j}=\psi_{j}^{\dagger} \psi_{j}$ is the fermion number at site $j$.

$$
\begin{aligned}
H_{A 1}= & -\frac{J_{1}}{2} \sum_{n}\left(\psi_{n+1}^{\dagger} \psi_{n}+\psi_{n}^{\dagger} \psi_{n+1}\right) \\
& +J_{1} \Delta \sum_{n}\left(\psi_{n}^{\dagger} \psi_{n}-1 / 2\right)\left(\psi_{n+1}^{\dagger} \psi_{n+1}-1 / 2\right) \\
& -g \mu_{B} H \sum_{n}\left(\psi_{n}^{\dagger} \psi_{n}-1 / 2\right)
\end{aligned}
$$

$$
\begin{aligned}
H_{A 2}= & J_{2} \sum_{n}\left(\psi_{n+2}^{\dagger} \psi_{n}+\text { h.c. }\right)\left(\psi_{n+1}^{\dagger} \psi_{n+1}-1 / 2\right) \\
& +J_{2} \sum_{n}\left(\psi_{n}^{\dagger} \psi_{n}-1 / 2\right)\left(\psi_{n+2}^{\dagger} \psi_{n+2}-1 / 2\right)(.5)
\end{aligned}
$$

$$
\begin{aligned}
H_{A 3}= & -J_{2} \delta \sum_{n}(-1)^{n}\left(\psi_{n+2}^{\dagger} \psi_{n}+\text { h.c. }\right)\left(\psi_{n+1}^{\dagger} \psi_{n+1}-1 / 2\right) \\
& -J_{2} \delta \sum_{n}(-1)^{n}\left(\psi_{n}^{\dagger} \psi_{n}-1 / 2\right)\left(\psi_{n+2}^{\dagger} \psi_{n+2}-1 / 2() 6\right)
\end{aligned}
$$

There is a difference between the first term of Eq.4 with the first term of Eq.5 and Eq.6, in Eq.4 first term present the hopping whereas the first term in Eq.5 and Eq. 6 are presenting the four fermionic interaction. This difference arises due to the presence of an extra factor $e^{-i \pi n_{j+1}}$ in the string of Jordan-Wigner transformation for NNN exchange interactions.

Similarly one can also recast the spin-chain systems with $J_{1}$ dimerization into the spinless fermions. The (2) Hamiltonians are converted as follows: $H_{B 1}=H_{A 1}$, $H_{B 2}=H_{A 2}$ and

$$
\begin{aligned}
H_{B 3}= & \frac{J_{1}}{2} \delta \sum_{n}(-1)^{n}\left(\psi_{n+1}^{\dagger} \psi_{n}+\psi_{n}^{\dagger} \psi_{n+1}\right) \\
& -J_{1} \delta \Delta \sum_{n}(-1)^{n}\left(\psi_{n}^{\dagger} \psi_{n}-1 / 2\right)\left(\psi_{n+1}^{\dagger} \psi_{n+1}-1 /(2 \pi)\right)
\end{aligned}
$$


In order to study the continuum field theory of these Hamiltonians, we recast the spinless fermions operators in terms of field operators by this relation.

$$
\psi(x)=\left[e^{i k_{F} x} \psi_{R}(x)+e^{-i k_{F} x} \psi_{L}(x)\right]
$$

where $\psi_{R}(x)$ and $\psi_{L}(x)$ are describe the secondquantized fields of right- and left-moving fermions respectively. The spin-Peierls compound, in the absence of magnetic field, is Generally at half-filling. In the absence of magnetic field $(H=0), k_{F}= \pm \pi / 2$, but we are interested to study the systems in presence of magnetic field, so we keep Fermi momentum as arbitrary $k_{F}$. One can simply absorb the finite magnetization in a shift of field $\phi$ by $\phi=\tilde{\phi}-\pi m x$, where $m=<S_{z}>$. In presence of magnetic field Fermi momentum and magnetization $(m)$ are related by this relation, $k_{F}=\frac{\pi}{2}(1-2 m)$ [6, 21]. We want to express the fermionic fields in terms of bosonic field by this relation

$$
\psi_{r}(x)=\frac{U_{r}}{\sqrt{2 \pi \alpha}} e^{-i(r \phi(x)-\theta(x))}
$$

$r$ is denoting the chirality of the fermionic fields, right (1) or left movers $(-1)$. The operators $U_{r}$ are operators that commute with the bosonic field. $U_{r}$ of different species commute and $U_{r}$ of the same species anticommute. $\phi$ field corresponds to the quantum fluctuations (bosonic) of spin and $\theta$ is the dual field of $\phi$. They are related by this relation $\phi_{R}=\theta-\phi$ and $\phi_{L}=\theta+\phi$.

Using the standard machinery of continuum field theory $6,21,22,23$, we finally get the bosonized Hamiltonians as

$$
\begin{aligned}
H_{0}= & v_{0} \int_{o}^{L} \frac{d x}{2 \pi}\left\{\pi^{2}: \Pi^{2}:+:\left[\partial_{x} \phi(x)\right]^{2}:\right. \\
& +\frac{g_{1}}{\pi^{2}} \int d x:\left[\partial_{x} \phi_{L}(x)\right]^{2}:+:\left[\partial_{x} \phi_{R}(x)\right]^{2}: \\
& +\frac{g_{2}}{\pi^{2}} \int d x\left(\partial_{x} \phi_{L}(x)\right)\left(\partial_{x} \phi_{R}(x)\right)
\end{aligned}
$$

$H_{0}$ is the gapless Tomonoga-Luttinger liquid part of the Hamiltonian with $v_{0}=\sin _{F}$. The analytical expressions for $g_{1}$ and $g_{2}$ (related with the forward scattering of fermionic field) are the following.

$$
\begin{gathered}
g_{1}=2\left(\Delta-2 J_{2}\right) \sin ^{2} k_{F}+2 J_{2} \sin 2 k_{F}\left(\pi+\sin 2 k_{F}\right) \\
g_{2}=4\left(\Delta-2 J_{2}\right) \sin ^{2} k_{F}+4 J_{2} \sin ^{2} 2 k_{F}
\end{gathered}
$$

The expression for $g_{1}$ and $g_{2}$ are the same of Ref. [24] and Ref. [6] at $m=0\left(k_{F}=\pi / 2\right)$.

Analytical expressions for different exchange interactions of Hamiltonian, $H_{A}$, are the following.

$H_{J 2 C 1}=\frac{J_{2}}{2 \pi^{2} \alpha^{2}} \int d x: \cos \left[4 \sqrt{K} \phi(x)-4 k_{F} x-4 k_{F} a\right]:$
$H_{J 2 C 2}=\frac{J_{1} \Delta}{2 \pi^{2} \alpha^{2}} \int d x: \cos \left[4 \sqrt{K} \phi(x)+4 k_{F} x-2 k_{F} a\right]:$.

$H_{J 2 C 3}=\frac{J_{2} \delta}{2 \pi^{2} \alpha^{2}} \int d x: \cos \left[\left(\pi-4 k_{F}\right) x+4 \sqrt{K} \phi(x)-4 k_{F} a\right]:$.

Eq.13 and Eq.14 are presenting the umklapp scattering term from the NN and NNN anti-ferromagnetic exchange interaction, Eq.15 is appearing due to the presence of dimerized interaction. Analytical expressions for $K$ is the following.

$K=\left[\frac{1-(8 / \pi) J_{2} \sin ^{2} k_{F}+4 J_{2} \cos _{F}}{1+(4 / \pi) \Delta \sin k_{F}+4 J_{2} \cos _{F}\left(1+2 / \pi \sin 2 k_{F}\right)}\right]^{1 / 2}$.

$v_{0}$ and $K$ are the two Luttinger liquid parameters. The expression of $\mathrm{K}$ for $J_{2}=0$ and $m=0$ is the same as in Ref. 21]. During this derivation we have used the following relations: $\rho_{R / L}=\frac{-1}{\pi} \partial_{x} \phi_{R / L}(x)$ and $[\phi(x), \Pi(x)]=i \delta\left(x-x^{\prime}\right)$, where $\Pi(x)=\frac{1}{\pi} \nabla \theta(x)$, is the canonically conjugate momentum. We have also used the following equations,

$$
S^{z}(x)=a\left[\rho(x)+(-1)^{j} M(x)\right] .
$$

The bosonized expressions for $\rho$ and $M$ are given by

$$
\begin{aligned}
\rho(x) & =-\frac{1}{\sqrt{\pi}} \partial_{x} \phi(x), \\
M(x) & =\frac{1}{\pi a} \cos (2 \phi(x)) .
\end{aligned}
$$

Similarly one can calculate the analytical expressions for $J_{1}$ dimerization. Totsuka 6] has already calculated the bosonized expressions for $J_{1}$ dimerization only by using the symmetric convention of chiral fermions $\left(, \psi_{L} \sim\right.$ $\left.e^{-2 i \phi_{L}}, \psi_{R} \sim e^{-2 i \phi_{R}}\right)$, and has expressed the sineGordon coupling, in terms of dual field $(\theta)$. Here we have expressed our all expressions in terms of bare phase field $(\phi)$, by using the conventional practice of continuum field theory $[21,22,23]$. During these derivations we assume that $J_{1} \gg J_{2}, \delta$. $J_{2}$ is in the unit of $J_{1}$. Here we neglect the higher order of $a$ than $a^{2}$.

\subsection{Calculations and Results for $m=0$ Magnetization Plateau:}

At first we discuss $m=0$ magnetization plateau, it corresponds $k_{F}= \pm \pi / 2$. Here we study both the effect of XXZ anisotropy $(\Delta)$ and the spin-Peierls dimerization $(\delta)$. The effective Hamiltonian for $J_{2}$ dimerization become,

$$
H_{A}=H_{0}+\left(\frac{J_{2}-\Delta}{2 \pi^{2} \alpha^{2}}\right) \int d x: \cos [4 \sqrt{K} \phi(x)]: .
$$

In this effective Hamiltonian (Eq.19), there is no contribution from dimerized interaction due to the oscillatory 
nature of the integrand and it leads to a vanishing contribution but the contribution of dimerized potential is present in the NN exchange interaction. Similarly the effective Hamiltonian for $J_{1}$ dimerization become,

$$
\begin{gathered}
H_{B}=H_{0}+\left(\frac{J_{2}-\Delta}{2 \pi^{2} \alpha^{2}}\right) \int d x: \cos [4 \sqrt{K} \phi(x)]: \\
+\frac{\delta}{2 \pi^{2} \alpha^{2}} \int d x: \cos [(2 \sqrt{K} \phi(x)]:
\end{gathered}
$$

This dimerization contribution for NN exchange interaction has originated from the XY interaction. The other two contribution of $J 1$ dimerization are from XXZ anisotropy of $\mathrm{NN}$ exchange interaction and z-component of NNN interaction. In recent past, there are considerable disagreement in conclusions. Chen et al. 25] claimed that in the limit of small frustration the spinPeierls dimerization in NNN exchange interaction destabilizes the isotropic Heisenberg fixed point, leading to a new gapless Luttinger liquid phase with vanishing spin wave velocity. Our study [20] has found that spin-Peierls dimerization in NNN exchange interaction present an irrelevant perturbation in the regime of small frustration. An explicit derivation of this $m=0$ plateau is relegated in the appendix. In this limit Parolla et al. [26] have also studied this model, and their conclusions are as the same as ours 20]. So it is a time relevant problem, present author would like to see this limit of plateau phase more explicitly. Now we would like to discuss this situation in the spinless fermions representation.

For the case of $J_{1}$ dimerization, we only consider the dispersion of free spinless fermions due to $J_{1}$, because the spin-Peierls perturbation will act only over the free spinless fermions due to $J_{1}$. The energy dispersion is $\epsilon_{k}=-J_{1} \cos k$, so in zero magnetic field, $k_{F}= \pm \pi / 2$. As a result wave vector $(Q=\pi)$ of dimerized potential is commensurate with the $2 k_{F}=\pi$ component of density wave and then it produce a gap in the excitation spectrum.

But the explanation for absence of, gapped state for $J_{2}$ dimerization is not so obvious. Only the intrinsic dimerization due to NNN exchange interaction can drive a system from gapless, spin-fluid, state to gapped dimer order state as one obtained from the Majumdar-Ghosh model [27]. Numerically it has predicted 28] for isotropic $(\Delta=1) J_{1}-J_{2}$ spin chain that the system drives from spin-liquid state to dimer order state for $J_{2}=0.241 J_{1}$. In our theoretical calculations, we predict this transition for $J_{2}=0.167 J_{1}$. Parola et al. have studied, numerically, the effect of $\delta$ on $J_{2}$ dimerization, there conclusion is negative, as we obtain analytically. It revealed from the variational study that the dimerization has only appreciable effect for higher values of NNN 20]. It is clear from Fig. 2A of our study that the NNN exchange interaction helps to produce a stable plateau phase for $J_{1}$ and $J_{2}$ dimerization, if one only consider the umklapp term due to the frustrating NNN exchange. Inset of Fig.

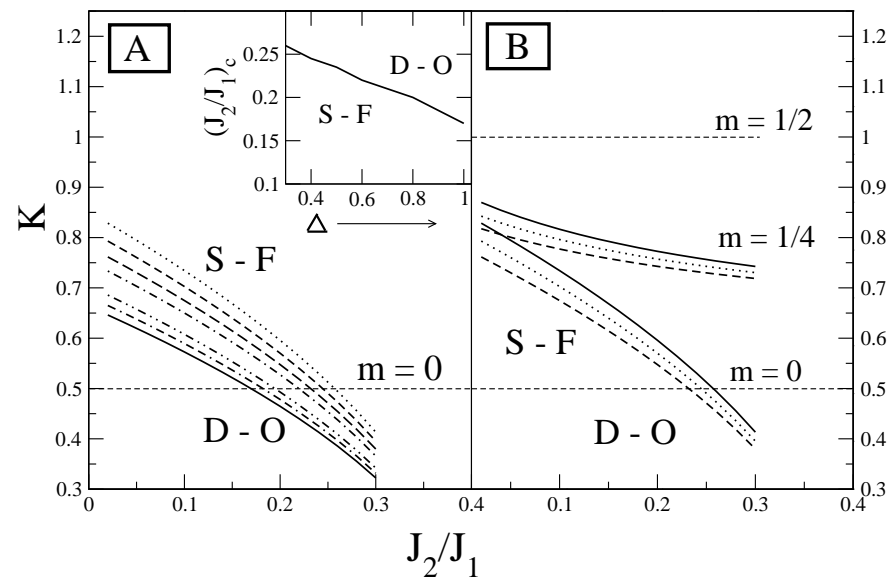

FIG. 2: Luttinger liquid parameter $(K)$ versus $\frac{J_{2}}{J_{1}}$ for different magnetization plateaus. A constant dashed line at $K=0.5$ is for eye guide line, which separating the spin fluid and dimer order instability state. D-O: dimer order instability state, SF: spin fluid Luttinger liquid phase. A: Here we only focus at $m=0$ magnetization plateau. Uppermost (dotted) curve is for $\Delta=0.3$ and the lowermost (solid) curve is for $\Delta=1$. The intermediate curves are for $\Delta=0.4,0.5,0.6 .0 .8,0.9$ respectively from upper to lower one. Inset shows the separation between the spin fluid and dimer order instability state by a critical line. Here we present the shift of the $\left(\frac{J_{2}}{J_{1}}\right)_{c}$ with $\Delta$. B: Here we present the curves for different magnetization plateaus. $m=1 / 2$ is independent of $\Delta$ and $\frac{J_{2}}{J_{1}}$ in contrast with $m=1 / 4,0$. The curves for $m=1 / 4,0$ plateaus are for $\Delta=0.4,0.5,0.6$ respectively from upper to lower one.

$2 \mathrm{~A}$, of our study present the variation of critical $\left(\frac{J_{2}}{J_{1}}\right)_{c}$ with the XXZ anisotropy. Below the critical line system display a short range anti-ferromagnetic order with gapless excitations by creating the low lying spinon states and above the critical line it is the gapped dimer order state. It reveals that lower values of $\Delta$ shift more the critical value of $\left(\frac{J_{2}}{J_{1}}\right)_{c}$ to change the phase from gapless spin-fluid phase to gapped dimer order phase. This is due to the enhancement of quantum fluctuation of the fields for lower $\Delta$, i.e., for the higher anisotropy.

It is also clear from the Fig $2 \mathrm{~A}$ and Fig. $2 \mathrm{~B}$ that the Luttinger liquid parameter $(K)$ is the function of $\Delta$ and $J_{2}$ and applied magnetic field. This dependence is modifying the anomalous scaling dimension of the physical field through $K$ and the commensurability properties of spin operator. So we conclude that the effect of $J_{2}$ dimerization (intrinsic dimerization) has pronounced effect near to the Heisenberg point $(\Delta=1$ and $K=1 / 2$ ). Spin gap is maximum at this point. In the dimerized phase this excitations are triplet with a gap in the excitation spectrum. One can conclude from the comparison of Fig. 2A (inset) of our study with the Fig. 4 of Ref. 26] that dimerization strength and XXZ anisotropy of NN interaction varying reciprocally, as it should be. Cross and Fisher have studied the spin-Peierls dimerization problem for $\mathrm{NN}$ anti-ferromagnetic spin 
chain [29]. They have predicted that spin gap $\propto(\delta J)^{2 / 3}$ . We also retrieve the same result on that special limit of our general problem. Actually on that limit spin gap is $\propto(\delta J)^{\frac{1}{2-K}}[29]$.

\subsection{Calculations and Results for $m=\frac{1}{4}$ Magnetization Plateau:}

Here we discuss the occurence of a finite (nontrivial) magnetization plateau We are considering the magnetization plateau at $m=\frac{1}{4}$, it corresponds $k_{F}= \pm \frac{\pi}{4}$. The effective Hamiltonian for $J_{2}$ dimerization become,

$$
\begin{aligned}
H_{A}= & H_{0}-\left(\frac{J_{2}+\delta}{2 \pi^{2} \alpha^{2}}\right) \int d x: \cos [4 \sqrt{K} \phi(x)]: \\
& +\frac{\Delta}{2 \pi^{2} \alpha^{2}} \int d x: \sin [4 \sqrt{K} \phi(x)]: .
\end{aligned}
$$

Apparently it appears from the general derivation of section (2), that the second and third terms of Eq. (21) will be absent due to the oscillatory nature of the integrand but this is not the case when one consider the dimerized lattice. In dimerized lattice, reciprocal lattice vector $G$ will change from $2 \pi$ to $\pi$ due to the change of the size of the unit cell. It become more clear, if one write these terms as $\int d x: \cos \left[\left(G-4 k_{F}\right) x+4 \sqrt{K} \phi(x)\right]:$.

Similarly one can write the effective Hamiltonian for $J_{1}$ dimerization:

$$
\begin{aligned}
H_{B}= & H_{0}-\frac{J_{2}}{2 \pi^{2} \alpha^{2}} \int d x: \cos [4 \sqrt{K} \phi(x)]: \\
& +\frac{\Delta(1+\delta)}{2 \pi^{2} \alpha^{2}} \int d x: \sin [4 \sqrt{K} \phi(x)]: .
\end{aligned}
$$

The analytical structure of Hamiltonian (Eq. 22 ) is the same as in Ref.6 for $J_{1}$ dimerization. The renormalization group equations for these type of interactions are 6., 21, 22, 30, 31].

$$
\begin{aligned}
& \frac{d K}{d \ln L}=-4 \pi^{2} K^{2} \delta^{2} \\
& \frac{d \delta}{d \ln L}=(2-4 K) \delta
\end{aligned}
$$

It appears from these RG equations that to get a relevant perturbation, $K$ should be less than $1 / 2$. It reveals from Fig. 3B that $K$ is exceeding the relevant value in our region of interest to mature criteria for magnetization plateau. So the dimerization strength should exceed some critical value $\left(\delta_{c}\right)$ to initiate the plateau phase. These two equations are the Kosterlitz-Thousless equation for the system in this limit. At the critical point, system undergoes Kosterlitz-Thouless transition 6, 21, 22, 30, 31]. Here we are not interested to present the renormalization group flow diagram because it has already discussed in Ref. [6]. The relation between the compactification radius $(R)$ of Ref. [6] and the Luttinger liquid parameter $(K)$ of our study is $K=R^{2}$. As we have mentioned that this phase diagram has already explored explicitly 6, 21, 22, 30, 31, 32], so we only present the relevant part of the phase diagram following Totsuka [6] and Tonegawa [8]: since the system flows to the strong coupling (dimer-order) as the dimerization strength exceeds some critical value $\left(\delta_{c}\right)$ initially, we have to guess the physics of this phase. We analyze the system in the limit $\delta \rightarrow \pm \infty$ and $K \rightarrow 0$. In this limit we can expect that the value of $\phi$ is pinned at one of the minima of $\cos (4 \sqrt{K} \phi)$. This parameter dependent transition, from massless phase to massive phase, at $T=0$ is the quantum phase transition 31. This quantum phase transition is occurring at the beginning and end of each magnetization plateau.

\subsection{Calculations and Results for $m=\frac{1}{2}$ Magnetization Plateau:}

Now we discuss the saturation plateau at $m=\frac{1}{2}$ $\left(k_{F}=0\right) . K_{F}=0$ implies that the band is empty and the dispersion is not linear, so the validity of the continuum field theory is questionable. Values of the two Luttinger liquid parameters, $v_{0}$ and $K$, are 0 and 1 respectively. It also implies that none of the sine-Gordon coupling terms become relevant in this parameter space. Saturation plateaus are only appearing due to very high values of magnetic field. In this plateau system is in ferromagnetic ground state and restore the lattice translational symmetry. We think, this is the classical phase of the system. Recently Cabra et al have studied the magnetization plateau for two leg ladder system with dimerized leg [10]. The configuration of leg dimerization is different from our present study, Fig. 1. In this configuration, each leg is dimerized by bond alternation and the two legs are connected by a rung coupling. In their study relevant operator arises from a combined effect of the interchain coupling and the dimerization along the chain. This plateau is the manifestation of applied potential not due to the higher values of magnetic field. This phase is the quantum phase of the system.

Here we present the possible explanation for the absence of other fractionally quantized magnetization plateaus (like $\frac{1}{3}, \frac{1}{5}$ etc): A carefull examination of Eq. 13 to Eq. 15 reveals that to get a nonoscillatory contribution from Hamiltonian one has to be satisfied $4 k_{F}=G$ condition but this condition is not fulfilled for these plateaus. The integrand of this sine-Gordon coupling terms contain an oscillatory factor that leads to a vanishing contribution. The other criteria is that non vanishing sine-Gordon coupling term should be relevant. This incident is same for both $J_{2}$ and $J_{1}$ dimerized chain. 


\section{DISCUSSIONS AND CONCLUSIONS:}

We have presented the generic feature of dimerized spin chain with $\mathrm{NN}$ and NNN exchange interaction under a magnetic field. Different quantized magnetization plateaus have occurred. The occurrence of $m=0$ magnetization plateau of $\mathrm{NN}$ exchange is spontaneous, whereas for NNN exchange interaction is not spontaneous. We have also predicted a phase transition line, which separate gapless spin-fluid phase to gapped dimer order state as a function of $\frac{J_{2}}{J_{1}}$ and $\Delta$. We expect that this critical line is the same for $J_{1}$ and $J_{2}$ dimerization, if one only consider the dimerized phase from intrinsic umklapp term. We also retrieve the result of Cross and Fisher [29] in one special limit of our general consideration. The basic origin of $m=\frac{1}{4}$ magnetization plateaus are the same for $J_{1}$ and $J_{2}$ dimerization because a critical strength of dimerization is needed to promote the state into a plateau phase. A critical strength of dimerized potential is necessary for the occurrence of this plateau in presence of NNN interaction. One can expect the Kosterlitz-Thouless type of transition for the occurrence of this plateau. This Kosterlitz-Thouless transition has been supported by the works of Totsuka [6] and Tonegawa [8]. Magnetization plateau at $m=\frac{1}{2}$ is the classical state of the system under high magnetic field. Cabra et al. 10. have predicted the $m=\frac{1}{2}$ magnetization plateau for zig-zag ladder with different leg dimerization than our present problem. This plateau is the quantum phase of the system. We have explained the absence of other fractionally quantized magnetization plateaus for our present problem. Magnetic field dependence response for a zig-zag ladder with modulation in exchange coupling have already studied by different groups numerically and they predict the existence of non zero magnetization plateau [33]. A higher order commensurabilities have occured for nonzero magnetization plateaus. This transition from commensurate gapped phase to gapless Luttinger liquid phase is the Mott- $\delta$ type of transition.

Author would like to acknowledge Prof. T. Giamarchi for several extensive discussions during the progress of this work and also Prof. B. I. Halperin, Prof. Dr. A. Honecker, and Prof. Diptiman Sen for partial discussion. Dr. Abhishek Dhar and Dr. Nandan Pakhira are deserving acknowledgement for reading the manuscript very critically. Finally author would like to acknowledge Prof. A. M. Finkelstein and Dr. Yuval Oreg for many useful discussions on low dimensional many body systems and also for warm support.

\section{APPENDIX}

In this appendix we present the explicit bosonization derivation for $m=0$ plateau of $J_{2}$ dimerization. Our starting Hamiltonian is

$$
H_{2 \delta}=-J_{2} \delta \sum_{n}(-1)^{n} \vec{S}_{n} \cdot \vec{S}_{n+2} .
$$

After Jordan-Wigner transformation ( Eq 3), Hamiltonian reduce to

$$
\begin{aligned}
H_{2 \delta}= & -J_{2} \delta \sum_{n}(-1)^{n}\left(\psi_{n+2}^{\dagger} \psi_{n}+\text { h.c. }\right)\left(\psi_{n+1}^{\dagger} \psi_{n+1}-1 / 2\right) \\
& -J_{2} \delta \sum_{n}(-1)^{n}\left(\psi_{n}^{\dagger} \psi_{n}-1 / 2\right)\left(\psi_{n+2}^{\dagger} \psi_{n+2}-1 / 2\right) .
\end{aligned}
$$

We linearize the energy spectrum around the Fermi points $k_{F}= \pm \frac{\pi}{2}$, and get the Hamiltonian in a final form

$$
\begin{aligned}
H_{2 \delta}= & -J_{2} \delta a^{2} \sum_{n}(-1)^{n}\left[-\left(\rho_{n+1}-(-1)^{n} M_{n+1}\right) \times\right. \\
& \left(\rho_{n}+\rho_{n+2}+(-1)^{n} M_{n}+(-1)^{n} M_{n+2}\right) \\
& \left.+\left(\rho_{n}+(-1)^{n} M_{n}\right)\left(\rho_{n+2}+(-1)^{n} M_{n+2}\right)\right] . \quad(27)
\end{aligned}
$$

During this derivation of $H_{2 \delta}$, we have used the following relations.

$$
\begin{aligned}
\rho(x) & =-\frac{1}{\sqrt{\pi}} \partial_{x} \phi(x), \\
M(x) & =\frac{1}{\pi a} \cos (2 \phi(x)) .
\end{aligned}
$$

Also, we have used Taylor expressions such as

$(R / L)(n+2)=(R / L)(n)+2 a\left(R^{\prime} / L^{\prime}\right)(n)+2 a^{2}\left(R^{\prime \prime} / L^{\prime \prime}\right)(n)+\cdots$

to write

$$
\begin{aligned}
& (R / L)^{\dagger}(n+2)(R / L)(n)+(R / L)^{\dagger}(n)(R / L)(n+2) \\
& =(R / L)^{\dagger}(n+2)(R / L)(n+2)+(R / L)^{\dagger}(n)(R / L)(n)+O(d 30)
\end{aligned}
$$

Where $R$ and $L$ are second quantized field of right and left moving fermions respectively. The non-oscillatory contributions of the Hamiltonian for $\mathrm{XY}$ and $\mathrm{Z}$ component of interaction are

$H_{2 \delta}(X Y)=J_{2} \delta a^{2} \sum_{n}\left[\rho_{n+1}\left(M_{n}+M_{n+2}\right)-M_{n+1}\left(\rho_{n}+\rho_{n+2}\right)\right]$.

$$
H_{2 \delta}(Z)=-J_{2} \delta a^{2} \sum_{n}\left[\rho_{n} M_{n+2}+M_{n} \rho_{n+2}\right] .
$$

Now we perform an operator product expansion of the above Hamiltonian. In the limit $z \rightarrow w$, we can use the following expansion [34],

$$
\begin{aligned}
\partial_{z} \phi(z): e^{i \beta \phi(w)}:= & -\frac{i \beta}{z-w}: e^{i \beta \phi(w)}: \\
& +: \partial_{z} \phi(z) e^{i \beta \phi(z)}:
\end{aligned}
$$


For our case $\beta=2$ and $K$ varies from less than $1 / 2$ to greater than $1 / 2$ depending on $\Delta$ and $\frac{J_{2}}{J_{1}}$ ratio. Here the values of $K$ are irrelevant because the various relevant terms in $H_{2 \delta}(X Y)$ and $H_{2 \delta}(Z)$ cancel each other due to the denominator, $z-w$, which takes values $\pm a, \pm 2 a$.

Apart from this detail mathematical analysis one can also understand the absence of this term from the following consideration: Here the applied dimerized potential is staggered. The sine-Gordon coupling term due to this perturbation is

$\frac{J_{2} \delta}{2 \pi^{2} \alpha^{2}} \int d x: \cos \left[\left(\pi-4 k_{F}\right) x+4 \sqrt{K} \phi(x)-4 k_{F} a\right]:$.

So at $k_{F}=\frac{\pi}{2}$ contribute a oscillatory term in the integrand and it leads to the vanishing contribution.

[1] F. D. M. Haldane, Phys. Rev. Lett 50, 1153 (1983) and Phys. Lett. A 93, 464 (1983); I. Affleck, in Fields, Strings and Critical Phenomena, ed. E. Brezin and J. Zinn-Justin (North-Holland, Amsterdam, 1989).

[2] J. G. Bednorz and K. A. Muller, Z. Phys. B 64, 188 (1986).

[3] D. C. Johnston, J. W. Johnson, D. P. Goshorn and A. J. Jacobson, Phys. Rev. B 35, 219 (1987); Z. Hiroi, M. Azuma, M. Takano and Y. Bando, J. Solid State Chem. 95, 230 (1991).

[4] R. Chitra and T. Giamarchi, Phys. Rev. B 55, 5816 (1997).

[5] M. Oshikawa, M. Yamanka, and I. Affleck, Phys. Rev. Letters 78, 1984 (1997).

[6] K. Totsuka, Phys. Rev. B 57, 3453 (1998); K. Totsuka, Phys. Letters. A 228, 103 (1997).

[7] W. Shiramura, K. Takatsu, H. Tanaka, K. Kamishima, M. Takahashi, H. Mitamura, and T. Goto, J. Phys. Soc. Jpn 66, 1900 (1997); W. Shiramura, K. Takatsu, B. Kurniawan, H. tanaka, H. Uekusa, Y. Ohashi, K. Takizawa H. Mitamura, and T. Goto, J. Phys. Soc. Jpn 67, 1548 (1998); T. Tonegawa, T. Nakao, and M. Kaburagi, J. Phys. Soc. Jpn 65, 3317 (1996).

[8] T. Tonegawa, T. Nishida, and M. Kaburagi, cond-mat/9712297

[9] H. J. Mikeska, and A. K. Kolezhuk in Quantum Magnetism, edited by U. Schollwock, J. Richter, D. J. J. Fernell, R. F. Bishop, Lecture Notes in Physics 645 (Springer, Heidelberg, 2004).

[10] D. C. Cabra, A. Honecker, and P. Pujol, Eur. Phys. J. B 13, 55 (2000); D. C. Cabra, A. Honecker, and P. Pujol, Phys. Rev. B 58, 6241 (1998); A. Honecker, Habil Thesis (private communication).

[11] A. Fledderjohann, C. Gerhardt, M. Karbach, K. H. Mutter, and R. wie $\beta$ ner, Phys. Rev. B 59991 (1999); R. wie $\beta$ ner, A. Fledderjohann, K. H. Mutter, and M. Karbach Eur. Phys. J. B 15, 475 (2000).
[12] K. Okunishi, Y. Hieida, and Y. Akutsu, Phys. Rev. B 60 R6953 (1999); A. Kawaguchi , Y. Fujii, and N. Kawakami, Phys. Rev. B 63144413 (2001); T. Yamamoto, R. Manago, Y. Mori, and C. Ishii, cond-mat/0204588

[13] R. Sato, and Y. Akatsu, J. Phys. Soc. Jpn 65, 1885 (1996).

[14] E. Dagotto, J. Riera and D. Scalapino, Phys. Rev. B 45, 5744 (1992); E. Dagotto and T. M. Rice, Science 271, 618 (1996).

[15] G. Chaboussant, M.-H. Julien, Y. Fagot-Revurat, L. P. Levy, C. Berthier, M. Horvatic, and O. Piovesana, Phys. Rev. Lett. 79, 925 (1997).

[16] S. Eggert, Phys. Rev. B 54, R9612 (1996); K. Okamoto and K. Nomura, Phys. lett. A 169, 433 (1992); R. Jullien and F. D. M. Haldane, Bull. Am. Phys. Soc. 28, 344 (1983); S. R. White and I. Affleck, Phys. Rev. B 54, $9862(1996)$.

[17] G. I. Japaridze, and A. A. Nersesyan, JETP Lett 27, 334 (1978).

[18] V. L. Pokrovsky, and A. L. Talapov, Phys. Rev. Lett. 42, 65 (1979).

[19] J. B. Parkinson, J. Phys. Condens. Matter 1, 6709 (1989).

[20] S. Sarkar and D. Sen, Phys. Rev. B 65, 172408 (2002).

[21] T. Giamarchi, Quantum Physics in One Dimension (Oxford Science Publications, Clarendon Press, Oxford, 2004).

[22] A. O. Gogolin, A. A. Nersesyan, and A. M. Tsvelik, Bosonization and Strongly Correlated Systems (Cambridge University Press, Cambridge, 1998).

[23] S. Rao and D. Sen in, Field Theory in Condensed Matter Physics, edited by S. Rao (Hindustan Book Agency, New Delhi, 2003); D. Senechal, cond-mat/9908262

[24] F. D. M. Haldane, Phys. Rev. B 25, 4925 (1982); 26, 5257 (E) (1982).

[25] S. Chen, H. Büttner, and J. Voit, Phys. Rev. Lett. 87, 087205 (2001); ibid 89, 149702 (2002); Phys. Rev. B 67, 054412 (20013).

[26] L. Capriotti, F. Becca, S. Sorella, and A. Parola, Phys. Rev. B 67172404 (2003); Phys. Rev. Lett. 89149701 (2002).

[27] C. K. Majumdar and D. K. Ghosh, J. Math. Phys. 10, 1388 and 1399 (1969).

[28] R. Chitra, S.Pati, H. R. Krishnamurthy, D. Sen, and S. Ramsesha, Phys. Rev. B, 52, 6581 (1995).

[29] M. C. Cross and D. S. Fisher, Phys. Rev. B 19, 402 (1979).

[30] J. M. Kosterlitz, and D. M. Thouless, J. Phys. C 6, 1181 (1973); V. L. Berezinski, Sov. Phys. JETP 32, 493 (1971).

[31] Subir Sachdev, Quantum Phase Transition (Cambridge University Press, Cambridge, 1998).

[32] S. Sarkar, Europhys. Lett 71, 980 (2005).

[33] M. Usami, and S. Suga, Phys. Lett. A 240, 85 (1998) and references therein.

[34] N. Nagaosa, Quantum Field Theory in Strongly Correlated Electronic Systems (Springer-Verlag, Berlin, 1999); C. J. Efthimiou and D. A. Spector, hep-th/0003190 